\title{
Metamaterials: electromagnetic enhancement at zero-index transition
}

\author{
Natalia M. Litchinitser, ${ }^{1, *}$ Andrei I. Maimistov, ${ }^{2}$ Ildar R. Gabitov, ${ }^{3}$ Roald Z. Sagdeev, ${ }^{4}$ and \\ Vladimir M. Shalaev \\ ${ }^{1}$ Department of Electrical Engineering, The State University of New York at Buffalo, Buffalo, \\ New York 14260, USA \\ ${ }^{2}$ Department of Solid State Physics, Moscow Engineering Physics Institute, Moscow 115409, Russia \\ ${ }^{3}$ Department of Mathematics, University of Arizona, Tucson, Arizona 85721, USA \\ ${ }^{4}$ Physics Department, University of Maryland College Park, College Park, Maryland 20740, USA \\ ${ }^{5}$ School of Electrical and Computer Engineering and Birck Nanotechnology Center, Purdue University, \\ West Lafayette, Indiana 47907, USA \\ *Corresponding author: natashal@buffalo.edu
}

Received July 1, 2008; revised August 5, 2008; accepted August 22, 2008; posted August 29, 2008 (Doc. ID 98159); published October 13, 2008

Resonant enhancement of electromagnetic waves propagating at oblique incidence in metamaterials, with dielectric permittivity and magnetic permeability linearly changing from positive to negative values, has been predicted and theoretically studied. This effect occurs for both TE and TM polarizations near the point where a refractive index changes its sign. Our model elucidates the unique features of the resonant enhancement in "positive-to-negative transition" metamaterials for a broad frequency range from microwaves to optics. () 2008 Optical Society of America

OCIS codes: $160.3918,160.2710,260.5740$.

The emergence of metamaterials has given rise to many unique electromagnetic (EM) phenomena, including negative index of refraction, which has never been found in nature, and enhanced interactions between materials and the magnetic field component of EM waves [1-4].

The unusual properties of negative-index materials (NIMs) are most prominently revealed at the interface of positive- and negative-index materials. Particularly, the right-handed triplet formed from the electric and magnetic fields and the wave vector $(E, H, k)$ in the positive-index material (PIM) undergoes an abrupt change to form a left-handed triplet in the NIM, revealing a topologically critical phenomenon that leads to antiparallel directions of the wave and Poynting vectors in the NIM.

To date, most of the studies in this area have been focused on abrupt transitions from PIMs to NIMs in homogeneous structures. However, recently it has been realized that inhomogeneous metamaterials open unparalleled opportunities for engineering space for wave propagation, thus enabling such fascinating functionalities as cloaking or wave concentrators [5-12]. In this Letter, we investigate the fundamental question of how EM waves propagate in an important class of inhomogeneous metamaterials, with material properties gradually changing from positive to negative values. We refer to these materials as transition metamaterials.

We discovered that resonant field enhancement occurs near the zero refractive index point under oblique incidence of the EM wave on a transition metamaterial layer. This phenomenon appears to have an analog in plasma physics, where it is referred to as resonant absorption [13-15]. We discuss several unique features of resonant EM enhancement in metamaterials, demonstrating that transition metamaterials provide a novel platform for fun- damental studies and potential applications of such EM enhancement phenomenon in a wide frequency range from microwaves to optical frequencies, with the important advantage of nearly unlimited flexibility in material parameter design.

We consider light propagation in a medium consisting of a homogeneous PIM in the region $x<0$ and a homogeneous NIM in the region $x>2 h$ separated by a transition layer of width $2 h$, where both $\epsilon$ and $\mu$ are real linear functions of the coordinate $x$ along the normal to the interface such that $\epsilon(x)=\epsilon_{0}(1-x / h)$ and $\mu(x)=\mu_{0}(1-x / h)$ if $0<x<2 h$, as shown in Fig. 1 (where $\mathrm{s} \equiv x / h$ ). For simplicity, we neglect herein realistic material losses and Fresnel reflections at the interfaces of the homogeneous and inhomogeneous regions.

The wave equation for the TE wave ( $E$ is perpendicular to the plane of propagation) is given by

$$
\frac{\partial^{2} E_{y}}{\partial x^{2}}+\frac{\partial^{2} E_{y}}{\partial z^{2}}-\frac{1}{\mu} \frac{\partial \mu}{\partial x} \frac{\partial E_{y}}{\partial x}+\frac{\omega^{2}}{c^{2}} \epsilon \mu E_{y}=0
$$

where $E_{y}$ is the amplitude of the electric component of the harmonic EM wave at frequency $\omega$ and $c$ is the

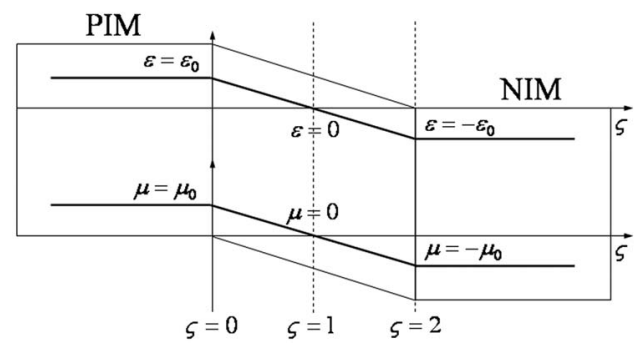

Fig. 1. Schematic of a transition layer between a homogeneous PIM layer and a homogeneous NIM layer. Dielectric permittivity and magnetic permeability in the transition region are real linear functions of the normalized coordinate $\mathrm{s}$. 
speed of light in vacuum. The components of the magnetic field are related to $E_{y}$ through the wellknown Maxwell's equations [13,14]. The corresponding equation for the TM wave can be obtained from Eq. (1) by replacing $E$ with $-H$, and $\mu$ with $\epsilon$, respectively.

Assuming that the medium is homogeneous in the $z$ direction, the electric field component can be written as $E_{y}=\Phi(x) \exp (i \beta z)$. Then Eq. (1) in the range $0<\varsigma<2$ takes the following form:

$$
\frac{\partial^{2} \Phi}{\partial \varsigma^{2}}+\frac{1}{(1-\varsigma)} \frac{\partial \Phi}{\partial \varsigma}+\left(a^{2}(1-\varsigma)^{2}-b^{2}\right) \Phi=0 .
$$

Here, $a=k_{0} h, k_{0}=\left(\epsilon_{0} \mu_{0}\right)^{1 / 2} \omega / c, b=\beta h, \beta=k_{0} \sin \left(\theta_{0}\right)$, and $\theta_{0}$ is the incidence angle.

While no unusual behavior of the electric and magnetic field components was found at normal incidence $(b=0)$, wave propagation at oblique incidence $(b \neq 0)$ turned out to be far more surprising. In this case, Eq. (2) indicates that when $a^{2}(1-\varsigma)^{2}-b^{2}<0$, incoming waves become evanescent. Note that there are two so-called "reflection points" at $s_{ \pm}=1 \pm b / a$ that are symmetric with respect to $s=1$. This makes the case under consideration different from that of propagation of an EM wave through a plasma layer, where there is only one such reflection point, as discussed in $[13,14]$. form

A general solution of Eq. (2) can be written in the

$$
\begin{aligned}
\Phi(\mathrm{s})= & C_{1} \exp \left(-i a(1-\varsigma)^{2} / 2\right) i a(1-\varsigma)^{2} \\
& \times U\left(1-i b^{2} / 4 a, 2, i a(1-\varsigma)^{2}\right)+C_{2} \\
& \times \exp \left(i a(1-\varsigma)^{2} / 2\right) i a(1-\varsigma)^{2} U\left(1+i b^{2} / 4 a, 2,\right. \\
& \left.-i a(1-\varsigma)^{2}\right),
\end{aligned}
$$

where $U$ is a confluent hypergeometric function. The coefficients $C_{1}$ and $C_{2}$ in Eq. (3) can be determined from boundary conditions.

To understand the behavior of the EM field near the point where both $\mu$ and $\epsilon$ change sign, we expand the solution (3) in the vicinity of $s=1$ and obtain

$$
\begin{aligned}
\Phi(\mathrm{s}) \approx & \left(C_{1}-C_{2}\right)\left(1+\frac{b^{2}(1-\varsigma)^{2}}{2} \ln \sqrt{a}(1-\varsigma)\right) \\
& -\frac{i}{2}\left(C_{1}+C_{2}\right)\left(1-\frac{\pi b^{2}}{4 a}\right) a(1-\varsigma)^{2} .
\end{aligned}
$$

The first term in this expression has a logarithmic singularity. The second term corresponds to a regular solution of Eq. (2). The logarithmic term is well defined for $s<1$; however, for $s>1$, the definition of the logarithm is not unique and depends on the choice of the path around $s=1$. Using Eq. (4) and following the approach used in [14], for the field components $E_{y}$, $H_{x}$, and $H_{z}$ near the point $\varsigma=1$ we obtain

$$
E_{y}=E_{0} \exp (i \beta z-i \omega t)+O\left((1-\varsigma)^{2}\right),
$$

$$
\begin{aligned}
H_{x}= & -E_{0} \sqrt{\epsilon_{0} / \mu_{0}}(1-\varsigma)^{-1} \sin \theta_{0} \exp (i \beta z-i \omega t) \\
& +O\left((1-\varsigma)^{2}\right), \\
H_{z}= & 2 i \pi E_{0} \epsilon_{0}(h / \lambda) \sin ^{2} \theta_{0} \ln \sqrt{a}(1-\varsigma) \exp (i \beta z-i \omega t) \\
+ & O\left((1-\varsigma)^{2}\right),
\end{aligned}
$$

where $E_{0}$ is the electric field amplitude at $\varsigma=1$. While the $y$ component of the electric field $E_{y}$ is continuous, the $x$ component of the magnetic field $H_{x}$ is singular at $\varsigma=1$, and the $z$ component $H_{z}$ experiences a jump when the value of $\mu$ changes sign from positive to negative at the point $s=1$. Following [14], we find that the difference of the longitudinal components of the Poynting vector $S_{x}=(c / 4 \pi) E_{y} H_{z}$ (averaged over rapid field oscillations) before and after the transition of the wave through the point $s=1$ is given by $\Delta S_{x}=c \pi E_{0}^{2}(h / \lambda) \epsilon_{0} \sin ^{2} \theta_{0}$. Note that while in our original model we assumed losses (imaginary parts of $\epsilon$ and $\mu$ ) to be infinitesimally small, at the point $\varsigma=1$ the real parts of $\epsilon$ and $\mu$ are zero; therefore, the contribution of the small imaginary parts of $\epsilon$ and $\mu$ becomes significant and can no longer be neglected. Next, we calculate the dissipation of energy $\Delta Q$ due to these losses at $\varsigma=1$ and find that $\Delta Q=\Delta S_{x}$. Therefore, transition through the point where $\epsilon$ and $\mu$ change sign even with infinitesimal loss is accompanied by a finite dissipation of incident wave energy. The specific mechanism of dissipation, determined by a physical model of a metamaterial, and the influence of the microscopic structure of realistic metamaterials on the field enhancement effect will be discussed elsewhere.

Figure 2 illustrates the real part of the electric field component $E_{y}$ as a function of a longitudinal coordinate $s$ for a fixed angle of incidence and fixed width of the transition layer. In numerical simulations, the spatially dependent material parameters $\epsilon$ and $\mu$ were taken in the form $\epsilon(\mathrm{s})=\epsilon_{0}(-\tanh [(\mathrm{s}-1) / l]+i \delta)$ and $\mu(\mathrm{s})=\mu_{0}(-\tanh [(\mathrm{s}-1) / l]+i \delta)$, where small loss parameter $\delta=0.00001$ was introduced to avoid the numerical complications around the point where the real part of $\mu$ approaches zero $(s=1)$ and $\epsilon_{0}=\mu_{0}=4$. Figure 3 shows the absolute value of the magnetic field component $H_{x}$ versus s, confirming the predic-

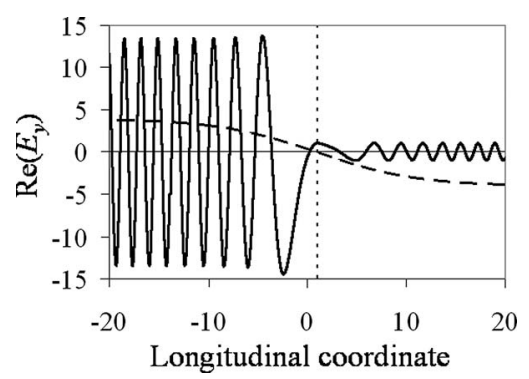

Fig. 2. Real part of the electric field component $E_{y}$ as a function of longitudinal coordinate $s$ for the case of oblique incidence at $\theta=\pi / 17$ (solid curve), the real part of the magnetic susceptibility $\mu$ as a function of longitudinal coordinate s (long-dashed curve), and the boundary between the PIM and NIM (short-dashed line). 


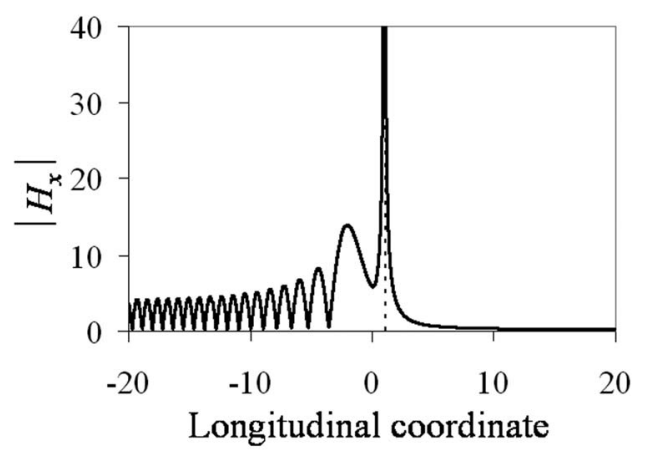

Fig. 3. Absolute value of the normalized magnetic field component $H_{x}$ as a function of longitudinal coordinate $s$ for the case of oblique incidence at $\theta=\pi / 17$.

tion that $H_{x}$ tends to infinity at $s=1$. While we have not assumed any specific structure of the metamaterial, in a simplified way the origin of the anomalous field enhancement shown in Fig. 3 can be understood as a spatial analog of the well-known resonance occurring in a spectral domain when light interacts with a harmonic oscillator [13]. In the case of the TM wave, the thin layer near the $\epsilon=0$ point can be considered as a very thin capacitor that accumulates infinitely large electric field energy if we neglect the effects of dissipation and spatial dispersion. Note that such energy accumulation occurs only for obliquely incident waves, since the electric field at the oblique incidence has a nonzero component in the direction of propagation. Since electric displacement $D$ must be continuous, the electric field $E$ anomalously increases as $\epsilon$ tends to zero. Likewise, for the TE wave considered herein, the magnetic field has a nonzero component in the direction of propagation, and the magnetic field energy accumulates in the vicinity of the $\mu=0$ point in space. Such a thin layer near the $\mu=0$ point can be considered as a short solenoid that stores the magnetic field energy. In this case, $H$ anomalously increases as $\mu$ tends to zero. Finally, owing to the singularities of the magnetic field components, the $x$ and $z$ components of the Poynting vector are also singular at $\mathrm{s}=1$. In addition, the $z$ component of the Poynting vector, given by $S_{z}=$ $-(c / 4 \pi) E_{y} H_{x}$, changes sign while passing the point $s$ $=1$. Although we considered only the case of the TE wave in this paper, similar effects can be demonstrated for the TM-wave case.

Interestingly, the phenomenon of resonant field enhancement and energy dissipation at a resonant point also occurs in inhomogeneous plasma with the dielectric permittivity changing as a function of the longitudinal coordinate [13-15]. However, in the case of plasma, the field enhancement near the point where $\epsilon=0$ occurs only in the TM-wave case, while in metamaterials we predicted resonant enhancement for both polarizations. Also, in inhomogeneous plasma with linearly changing $\epsilon$, there is a single "reflection" point and no propagating waves are supported past the resonance point (where $\epsilon<0$ ), whereas in metamaterials there are two reflection points and waves are allowed to propagate beyond the resonance point in the negative refractive index region. These unique features of the resonant EM enhancement effect in metamaterials are made possible by the fundamentally new feature facilitated by metamaterials-enhanced interaction of the magnetic field component of the EM wave with the material. As a result, the dynamics of wave propagation in inhomogeneous metamaterials as compared with that in plasma is more elaborate, suggesting that metamaterials provide a superior environment for further studies of fundamental and applied aspects of resonant effects in inhomogeneous media.

In summary, our study reveals an important new phenomenon occurring upon the EM wave propagation in inhomogeneous metamaterials with material parameters linearly changing from positive to negative values: At oblique incidence, we analytically and numerically demonstrated the phenomenon of resonant EM enhancement in the vicinity of the point where $\epsilon$ and $\mu$ change signs. This finding is likely to enable a variety of applications in microwave, terahertz, and optical metamaterials, including subwavelength transmission, antennas, and low-intensity nonlinear optical devices.

The authors thank V. G. Veselago, J. B. Pendry, A. C. Newell, R. W. Boyd, and G. Shvets for enlightening discussions. This research was supported by the United States Army Research Office (USARO) through grants W911NF-07-1-034 and 50342-PHMUR, the National Science Foundation (NSF) through grant DMS-050989, and the Russian Foundation for Basic Research (RFBR) through grant 06-02-16406.

\section{References}

1. V. G. Veselago, Sov. Phys. Usp. 10, 509 (1968).

2. J. B. Pendry, Phys. Rev. Lett. 85, 3966(4) (2000).

3. V. M. Shalaev, Nat. Photonics 1, 41 (2007).

4. N. M. Litchinitser, I. R. Gabitov, A. I. Maimistov, and V. M. Shalaev, in Progress in Optics, E. Wolf, ed. (Elsevier, 2008), Vol. 51, pp. 1-68.

5. M. Rahm, D. Schurig, D. A. Roberts, S. A. Cummer, D. R. Smith, and J. B. Pendry, Photonics Nanostruct. Fundam. Appl. 6, 87 (2008).

6. A. V. Kildishev and V. M. Shalaev, Opt. Lett. 33, 43 (2008).

7. M. Rahm, S. A. Cummer, D. Schurig, J. B. Pendry, and D. R. Smith, Phys. Rev. Lett. 100, 063903(4) (2008).

8. J. B. Pendry, D. Schurig, and D. R. Smith, Science 312, 1780 (2006).

9. U. Leonhardt, Science 312, 1777 (2006).

10. D. Schurig, J. J. Mock, B. J. Justice, S. A. Cummer, J. B. Pendry, A. F. Starr, D. R. Smith, Science 314, 977 (2006).

11. W. Cai, U. K. Chettiar, A. V. Kildishev, and V. M. Shalaev, Nat. Photonics 1, 224 (2007).

12. W. Cai, U. K. Chettiar, A. V. Kildishev, G. W. Milton, and V. M. Shalaev, Appl. Phys. Lett. 91, 111105(3) (2007).

13. V. L. Ginzburg, The Propagation of Electromagnetic Waves in Plasma (Pergamon, 1970).

14. L. D. Landau, E. M. Lifshitz, and L. P. Pitaevskii, Electrodynamics of Continuous Media, 2nd ed. (Butterworth-Heinemann, 1984), Vol. 8.

15. A. O. Korotkevich, A. C. Newell, and V. E. Zakharov, J. Appl. Phys. 102, 083305(14) (2007). 